\title{
Problématique de l'irrigation sur l'exploitation
}

\author{
L. Rieul \\ Délégué du secteur hydraulique agricole du RNED \\ Division Irrigation du CEMAGREF - Montpellier
}

\section{Introduction}

Les problèmes à résoudre par l'exploitant agricole pour bien conduire l'irrigation sur les parcelles de son exploitation ne sont pas simples. Il ne s'agit pas, dans la plupart des cas, d'apporter systématiquement de l'eau pour maintenir les cultures dans un confort hydrique permanent, en vue d'une production maximale. Pour optimiser sa production, l'irrigant doit définir la meilleure répartition dans l'espace et dans le temps du volume d'eau disponible.

Dans ce but il doit raisonner le choix de ses assolements et définir une stratégie d'irrigation compte tenu des besoins en eau des cultures mais aussi des contraintes de l'exploitation telles que le niveau et la nature des équipements d'irrigation, la main-d'œuvre disponible, etc... Il doit ensuite conduire et contrôler l'irrigation de manière à réaliser aussi bien que possible la stratégie choisie, ou l'adapter en cours de campagne si nécessaire.

\section{Choix d'une stratégie d'irrigation}

La stratégie d'irrigation doit être définie à l'échelle de l'exploitation en fonction des besoins en eau des cultures et des possibilités d'irrigation.

Les besoins en eau des cultures et leur sensibilité à la sécheresse sont relativement bien connus. Des fonctions globales liant le rendement des cultures au taux de satisfaction des besoins en eau ont été établies pour les principales cultures [1]. Ces fonctions doivent être améliorées pour tenir compte des sensibilités des cultures aux différents stades de leur cycle végétatif.

En effet l'INRA a mis en évidence des périodes critiques du cycle végétatif durant lesquelles les plantes présentent une sensibilité particulière à la sécheresse, plus ou moins grande suivant les espèces [2].
Il sera alors possible de tester les résultats que l'on peut obtenir sur une culture déterminée, avec des apports d'eau plus ou moins limités à différentes périodes. On pourra ainsi évaluer pour différentes cultures les risques et les conséquences dus à la sécheresse compte tenu des prévisions climatiques et des possibilités d'irrigation.

Les possibilités d'irrigation sont fonction de la ressource en eau disponible, de l'importance des réserves en eau du sol mais aussi des contraintes liées aux équipements d'irrigation et à la disponibilité de la main-d'auvre sur l'exploitation.

La gestion de la mobilisation de l'eau du sol diffère selon l'importance des réserves et la nature des ressources en eau disponibles (RIEUL, 1991).

On aura souvent intérêt à maintenir les réserves en eau du sol à un bon niveau en début de campagne d'irrigation, par des irrigations fréquentes, de manière à les mobiliser en période de besoin de pointe pour écrêter la pointe.

De même si l'on irrigue à partir d'une rivière ou d'une nappe alluviale à fort tarissement, on s'efforcera d'irriguer au maximum avant la période de tarissement, pour maintenir pleines les réserves du sol jusqu'à cette période.

Au contraire, si l'on dispose d'un volume limité que l'on peut mobiliser à n'importe quel moment (retenue collinaire), on peut avoir intérêt à laisser les cultures utiliser au maximum les réserves du sol en début de campagne, au risque d'un certain stress hydrique en début de période de végétation, de manière à réserver l'usage de l'eau d'irrigation aux moments critiques.

Les besoins des différentes cultures et les possibilités d'irrigation étant bien définis, il est possible de rechercher par simulation la combinaison optimale des cultures qui définira l'assolement et d'établir le calendrier d'irrigation correspondant (THÉVENET, 1990). Une telle optimisation est complexe et nécessite de bonnes références. Des logiciels ont été élaborés pour aider la collecte des données pour la gestion des parcelles et le choix des assolements. Ces logiciels ont fait l'objet de tests et d'analyse approfondie par un groupe de travail du RNED-HA, dont les résultats ont été publiés récemment [3]. 


\section{Conduite de l'irrigation}

Pour atteindre les objectifs qu'il s'est fixé, l'irrigant doit, en cours de campagne, piloter correctement l'irrigation et vérifier le fonctionnement de ses matériels.

Le pilotage de l'irrigation consiste à maîtriser la bonne adéquation des apports d'eau d'irrigation aux besoins réels des cultures, compte tenu de l'évolution climatique et agronomique et si nécessaire, à réajuster en conséquence la stratégie prévisionnelle.

Dans ce but, il faut suivre le bilan hydrique des parcelles de l'exploitation. Une source d'erreur fréquente du bilan hydrique réside dans la difficulté d'apprécier la contribution du sol à l'alimentation en eau des cultures, d'où souvent, la nécessité de contrôler le bilan par l'observation d'un indicateur physique de l'état hydrique.

L'outil de contrôle actuellement le plus opérationnel est le tensiomètre, qui permet de suivre l'évolution de l'état hydrique du sol à différentes profondeurs [4].

Des méthodes plus récentes sont basées sur la mesure d'un indicateur de stress hydrique de la plante : température de surface du couvert végétal [5]; micro-variations de diamètre des organes végétaux [6]. Ces méthodes permettent de gérer en temps réel le stress hydrique des cultures tout au long du cycle végétatif. Plus précises et moins souples que les méthodes classiques (bilan hydrique et tensiomètre), ces méthodes de pilotage en temps réel exigent un équipement d'irrigation fixe automatique et performant, qui puisse répondre rapidement aux besoins sur l'ensemble de la parcelle lorsque le seuil de stress est atteint [5].

La stratégie et le pilotage de l'irrigation définissent le moment auquel il faut déclencher l'irrigation et la quantité d'eau qu'il faut apporter. L'irrigant doit encore s'assurer les moyens de bien répartir la quantité d'eau nécessaire de manière aussi uniforme que possible et sans excès sur l'ensemble des parcelles irriguées.

L'utilisation et le fonctionnement des équipements conditionnent cette bonne répartition.

En irrigation de surface, l'évolution technique des équipements permet de mieux contrôler les apports d'eau sur la parcelle et de conduire plus efficacement l'irrigation, en diminuant de manière significative les consommations d'eau [7]. Cela exige un effort de réhabilitation des vieux réseaux et de formation des irrigants aux modes de conduite de l'irrigation avec des matériels modernes.

En irrigation sous pression, les équipements existants doivent permettre un bon contrôle des apports d'eau. Il faut néanmoins veiller à ce que les caractéristiques des matériels choisis, leur disposition et leur réglage au champ soient bien adaptés aux conditions réelles de fonctionnement. De récents diagnostics de contrôle de fonctionnement de telles installations ont montré que souvent les doses d'irrigation réellement apportées diffèrent sensiblement des apports souhaités du fait du mauvais réglage des appareils (enrouleurs), de mauvaises dispositions des arroseurs (asperseurs en zones ventées) ou de mauvaises conditions de fonctionnement (pression insuffisante) (DUBALEN, 1992).

De même de nombreux arboriculteurs, dans le Sud-Est, rencontrent des problèmes dans l'utilisation de l'irrigation localisée, notamment du fait de difficultés dans la filtration de l'eau. Cela les conduit dans certains cas à compléter la micro-irrigation par des aspersions ou des submersions (Le Doré et Revol, 1991).

Cela montre bien que le choix de matériels modernes et performants ne suffit pas à garantir l'efficacité de l'irrigation. Il faut que ces matériels soient bien adaptés au contexte de l'exploitation et à la stratégie d'irrigation choisie et qu'ils fonctionnent dans de bonnes conditions.

\section{Conclusion}

L'irrigation a connu au cours de ce siècle une évolution technique importante qui vise à la diminution des interventions manuelles pénibles et à une amélioration sensible de l'efficience de l'irrigation. Cette évolution ne concerne pas seulement les équipements de distribution de l'eau mais aussi les outils de pilotage de l'irrigation. La valorisation des innovations conduit l'exploitant à mieux raisonner la stratégie d'irrigation à l'échelle de l'exploitation et à gérer les moyens de réaliser cette stratégie. Ces évolutions font de l'irrigation, art très ancien, une véritable science que l'irrigant doit maîtriser de plus en plus.

Dans ce but des travaux de recherche doivent se poursuivre afin de mettre à disposition des références et des outils d'aide à la décision. Cela concerne notamment l'étude des matériels, la conduite de l'irrigation, l'optimisation technique et économique des choix. L'acquisition de références opérationnelles à travers l'opération secteurs de référence Irrigation complète les travaux de recherche. Enfin le secteur Hydraulique agricole du RNED, qui associe étroitement la recherche et le développement, s'efforce d'accélérer la diffusion des acquis vers le développement, notamment à travers ses publications [8].

\section{Bibliographie}

[1] Puech J., Marty J.R., Maertens C. (1976). - Efficience de l'eau consommée par divers végétaux et application à la valorisation de l'irrigation.

[2] Robelin M. (1963). - Contribution à l'étude du comportement du maïs grain vis-à-vis de la sécheresse ; journées nationales de l'irrigation - AGPM.

[3] RNED-HA (1992). - Des logiciels pour la gestion de parcelles, le choix d'assolement.

[4] Peyremorte P. et Isberie C. - Les tensiomètres - Maîtrisez votre irrigation - RNED 1986.

[5] Delacourt A., Panine M., Rieul L. (1989). - Expérimentations et exemples d'application des méthodes modernes de pilotage de l'irrigation en Languedoc-Roussillon - Colloque des Nations Unies à Avignon.

[6] Huguet J.G. (1986). - Appréciation de l'état hydrique d'une plante à partir des variations micrométriques de la dimension des fruits ou des tiges au cours de la journée. Agronomie $5 \mathrm{p}$. 733-741.

[7] RNED-HA (1989). — Modernisation de l'irrigation à la raie.

[8] RNED-HA (1992), - Guide pratique irrigation ( $2^{\mathrm{e}}$ édition). 